\title{
Direct Determination of Bromide, Nitrate, and lodide in Saline Matrixes Using Electrostatic Ion Chromatography with an Electrolyte as Eluent
}

\author{
Wenzhi Hu, ${ }^{,+\uparrow}$ Paul R. Haddad, ${ }^{*, \ddagger}$ Kyoshi Hasebe, ${ }^{\S}$ Kazuhiko Tanaka,, Philip Tong, ${ }^{\perp}$ and Cheang Khoo \\ Division of Chemistry, Graduate School of Science, Hokkaido University, Sapporo 060, Japan, School of Chemistry, \\ University of Tasmania, GPO Box 252-75, Hobart 7001, Australia, Division of Material Science, \\ Graduate School of Environmental Earth Science, Hokkaido University, Sapporo 060, Japan, \\ National Industrial Research Institute of Nagoya, 1-1 Hirate-cho, Kita-ku, Nagoya 462-8510, Japan, \\ Australian Government Analytical Laboratory, 1 Suakin Street, Pymble, NSW 2073, Australia, and Faculty of Informatics, \\ Science and Technology, University of Western Sydney, Macarthur, NSW 2560, Australia
}

A new ion chromatographic method that is applicable to the direct determination of UV-absorbing inorganic anions in saline matrixes is described. An octadecylsilica column modified with a zwitterionic surfactant (3-(N,N-dimethylmyristylammonio)propanesulfonate) is used as the stationary phase, and an electrolytic solution is used as the eluent. Under these conditions, the matrix species (such as chloride and sulfate) are only retained weakly and show little or no interference. It is proposed that a binary electrical double layer (EDL) is established by retention of the eluent cations on the negatively charged (sulfonate) functional groups of the zwitterionic surfactant (forming a cation-EDL) and by retention of eluent anions on the positively charged (quaternary ammonium) functional groups of the zwitterionic surfactant (forming an anion-E DL). Sample anions are able to distribute into the cation-EDL and to form ion pairs with the EDL cations, while at the same time experiencing repulsion from the anion-EDL. Anions are therefore eluted in order of increased propensity to form ion pairs. The method has been applied to the determination of bromide, nitrate, and iodide in artificial seawater, giving detection limits of $\mathbf{0 . 7 5}$ $\mathrm{ppb}$ for bromide, $0.52 \mathrm{ppb}$ for nitrate, and $0.8 \mathrm{ppb}$ for iodide using UV absorbance detection at $210 \mathrm{~nm}$ and relative standard deviations of $<1.2 \%$. Real seawater samples have also been analyzed successfully.

In a series of previous studies ${ }^{1-7}$ employing stationary phases coated with zwitterionic surfactants (i.e., those containing both positively and negatively charged functional groups but carrying no formal net charge), it has been demonstrated that inorganic anions can be separated using pure water as eluent, with unique separation selectivity. This method has been termed electrostatic

\footnotetext{
† Division of Chemistry, Hokkaido University.

‡ University of Tasmania.

$\S$ Division of M aterial Science, Hokkaido University.

"National Industrial Research Institute of Nagoya.

${ }^{\perp}$ Australian Government Analytical Laboratory.

University of Western Sydney.

(1) Hu, W.; Takeuchi, T.; Haraguchi, H. Anal. Chem. 1993, 65, 2204-2208.

(2) Hu, W.; Tao, H.; Haraguchi, H. Anal. Chem. 1994, 66, 765-767.
}

ion chromatography (EIC), and the separation has been attributed to a simultaneous electrostatic attraction and repulsion mechanism occurring at both the positive and the negative charges on the stationary phase. A drawback of EIC is that when the sample contains multiple anions and cations, each analyte anion may be eluted as more than one peak, with each peak being a specific combination of the anion with one of the cations of the sample. Recently, we showed ${ }^{8}$ that addition of a small quantity of a suitable electrolyte to the eluent causes analyte anions to be eluted only as a single peak, irrespective of the number and type of cations in the sample. In the present study, we have investigated the separation mechanism in more detail and have applied the method to the determination of nitrate, bromide, and iodide in seawater.

The determination of nitrate, bromide, and iodide in seawater is of importance to oceanographic research..$^{9-11}$ Ion-exchange chromatography (IEC) is generally inapplicable to this analysis for several reasons. First, the large amount of matrix ions (chloride, sulfate) saturate the active sites of the stationary phase and thereby impede the separation of the target analytes. Second, the high ionic strength of the sample causes self-elution of the sample band during injection, leading to peak broadening and loss of separation efficiency. Third, the levels of the target analytes are often very low so that detection becomes a major problem, especially when the eluted peaks are poorly defined in shape.

To overcome these difficulties, Ito and Sunahara ${ }^{12}$ suggested the use of the matrix ions as the eluent ions, for example, the use of relatively high concentrations of sodium chloride solution

(3) Hu, W.; M iyazaki, A.; Tao, H.; Itoh, A.; Umemura, T.; Haraguchi, H. Anal Chem. 1995, 67, 3713-3716.

(4) Hu, W.; Haraguchi, H. J. Chromatogr., A 1996, 723, 251-258.

(5) Hu, W.; Hasebe, K.; Reynols, D. M.; Umemura, T.; Kamiya, S.; Itoh, A.; Haraguchi, H. J. Liq. Chromatogr., Relat. Technol. 1997, 20, 1903-1919.

(6) Hu, W.; Haddad, P. R. Trends Anal. Chem. 1998, 17, 69-72.

(7) Hu, W.; Hasebe, K.; Reynolds, D. M.; Haraguchi, H. Anal. Chim. Acta 1997 $353,143-149$.

(8) Hu, W.; Haddad, P. R. Anal. Commun. 1998, 35, 317-320.

(9) Sigman, D. M .; Altabet, M. A.; M ichener, R.; M cCorkle, D. C.; Fry, B.; Holmes, R. M. Mar. Chem. 1997, 57, 227-242.

(10) Debaar, H. J. W.; Vanlecuwe, M. A.; Scharek, R.; Goeyens, L.; Bakker, M J.; Fritsche, P. Deep-Sea Res. 1997, 44, 229ff.

(11) Hutchins, D. A.; Bruland, K. W. Nature 1998, 393, 561-564.

(12) Ito, K.; Sunahara, H. Bunseki Kagaku 1988, 37, 292-295. 
as the eluent in the analysis of seawater. When this is done, the separation column becomes equilibrated with the matrix ions before the sample injection and the sample matrix ions will therefore show little or no retention on the stationary phase and interference effects are minimized. This method can be termed "matrix elimination ion chromatography" and nitrite and/or nitrate $\mathrm{e}^{13,14}$ and iodide ${ }^{15-18}$ in seawater have been determined successfully using this approach. However, some practical problems persist, including the requirement to use nonmetallic hardware to avoid corrosion, and an inability to separate nitrate, bromide and iodide in a single run.

\section{EXPERIMENTAL SECTION}

Apparatus. The HPLC system used throughout this study was a Shimadzu LC-10A system, consisting of a LC-10AT pump (Shimadzu, Kyoto, Japan), a sample injector (Rheodyne, Cotati, CA) with a 100- $\mu$ L loop, a CTO-10A column oven (Shimadzu), and an SPD-10A UV-visible detector (Shimadzu) used in tandem with a CDD-6A conductivity detector (Shimadzu). A CR-6A Chromatopac data system (Shimadzu) was used for recording the chromatograms. The column used throughout this study (L-column, $250 \times 4.6 \mathrm{~mm}$ i.d., Chemical Inspection and Testing Institute, Tokyo, Japan) was packed with ODS material which had been coated with 3-(N,N-dimethylmyristylammonio)propanesulfonate (Zwittergent-3-14) micelles. The procedure for the preparation of the zwitterionic surfactant-coating column has been described in previous papers. ${ }^{21,23}$

Reagents. The zwitterionic surfactant Zwittergent-3-14, used for column preparation, was obtained from Calbiochem ( La J olla, CA). Inorganic salts used to prepare the mobile phase and the samples were purchased from Wako (Osaka, Japan) and were used as received. Pure water used throughout this study was prepared in the laboratory using a Milli-Q purification system ( M illipore, B edford, M A). Artificial seawater was prepared using the method of Lyman and Fleming ${ }^{19}$ and contained the following ions: $\mathrm{Na}^{+}, \mathrm{K}^{+}, \mathrm{M} \mathrm{g}^{2+}, \mathrm{Ca}^{2+}, \mathrm{Cl}^{-}$, and $\mathrm{SO}_{4}{ }^{2-}$ at molalities of 0.4822 , $0.05489,0.01063,0.01062,0.5657$, and 0.02906 , respectively. Actual seawater samples were taken from the surface of the Japan Sea near the shore (Otaru, Hokkaido, Japan).

A standard sample was obtained by dissolving $\mathrm{NaBr}, \mathrm{NaNO}_{3}$, and $\mathrm{Nal}$ in the artificial seawater matrix to give concentrations of $\mathrm{Br}^{-}, \mathrm{NO}_{3}^{-}$, and $\mathrm{I}^{-}$of 1000,50 , and $5.0 \mathrm{ppb}$, respectively.

\section{RESULTS AND DISCUSSION}

Effect of Eluent Composition. Until recently, our previous EIC studies had been performed using pure water as the eluent.

(13) Ito, K.; Ariyoshi, Y.; Tanabiki, F.; Sunahara, H. Anal. Chem. 1991, 63, 273276.

(14) Rokushika, S.; Yamamoto, F. J. Chromatogr. 1993, 630, 195-200.

(15) Ito, K.; Shoto, E.; Sunahara, H. J. Chromatogr. 1991, 549, 265-272.

(16) M cTaggart, A. R.; Butler, E. C. V.; Haddad, P. R.; Middleton, J. H. Mar. Chem. 1994, 47, 159-172.

(17) Brandao, A. C. M .; Buchberger, W. W.; Butler, E. C. V.; Fagan, P. A.; Haddad, P. R. J. Chromatogr., A 1995, 706, 271-275.

(18) Ito, K. Anal. Chem. 1997, 69, 3628-3632.

(19) Lyman, J.; Fleming, R. H. Mar. Res. 1940, 3, 134.

(20) Bjerrum, N. K. Dan. Vidensk. Selsk. 1926, 7, 1.

(21) Johnson, K. S.; Pytkowicz, R. M. Am. J. Sci. 1978, 278, 1428.

(22) Kester, D. R.; Pytkowicz, R. M. Mar. Chem. 1975, 3, 365.

(23) Pytkowicz, M. R., Ed. Activity Coefficients in Electrolyte Solutions; CRC Press: Boca Raton, FL, 1979; Vol. II.

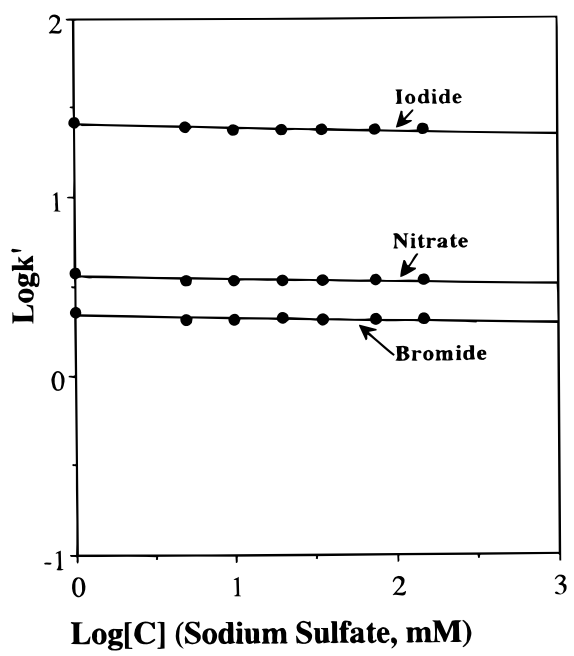

Figure 1. Log $K$ versus log [eluent] for a $\mathrm{Na}_{2} \mathrm{SO}_{4}$ eluent.

However, we reported recently in a preliminary communication ${ }^{8}$ that addition of a small amount ( $5.0 \mathrm{mM}$ ) of $\mathrm{NaHCO}_{3}$ to the eluent caused some major effects, namely, that analyte anions were eluted as a single peak regardless of the number and type of cations in the sample and that increases in the concentration of the eluent above $5.0 \mathrm{mM}$ had little further effect on retention times. One of the first aims of the present work was to explore the utility of further inorganic salts as eluent additives, such as sodium chloride, sodium sulfate, and sodium perchlorate. Because of our intention to apply the method to the analysis of high ionic strength samples such as seawater, nitrite, bromide, nitrate, and iodide were chosen as typical analyte species because of their status as important species in oceans and because they are amenable to sensitive direct VU - visible detection.

A standard sample containing 1000,50 , and $5.0 \mathrm{ppb}$ of $\mathrm{Br}^{-}$, $\mathrm{NO}_{3}^{-}$, and $\mathrm{I}^{-}$, respectively, made up in the artificial seawater matrix, was used to examine the effect of eluent composition. When a solution of a single ionic salt (sodium chloride, sodium sulfate, or sodium perchlorate) was used as eluent it was noted that the concentration of the electrolyte in the eluent did not exert a significant effect on analyte retention. This is illustrated in Figure 1 , which shows a plot of log $\mathrm{k}^{\prime}$ versus log [eluent] for a $\mathrm{Na}_{2} \mathrm{SO}_{4}$ eluent and the results are in agreement with our earlier observations using a $\mathrm{NaHCO}_{3}$ eluent. ${ }^{8}$ The void time for this column was found to be $1.80 \mathrm{~min}$, which is very close to the retention times for sulfate and chloride (determined in a separate experiment using conductivity detection ${ }^{8}$ ).

Next, serial dilutions of the artificial seawater were used as eluent for the separation of the same standard sample in order to explore the possibility of matrix elimination behavior in EIC. Retention factors for the target analytes were plotted as a function of the eluent concentration (because of the range of species in the eluent, $\log \left(C^{*}\right)$, where $C^{*}$ is the dilution factor, was used instead of log [eluent]. Here, a dilution factor of 100 implies a 100 -fold dilution of the artificial seawater). Figure 2 shows the results obtained and indicates that log $\mathrm{k}^{\prime}$ decreased almost linearly with increasing $\log \left(C^{*}\right)$ (the correlation coefficients were found to be $0.979,0.983$, and 0.991 for bromide, nitrate, and iodide, respectively). The values for the slope of the plot for $\mathrm{Br}^{-}, \mathrm{NO}_{3}{ }^{-}$, and $\mathrm{I}^{-}$were found to be $-0.131,-0.149$, and -0.165 , which are much smaller than the theoretical value of -1 expected for 


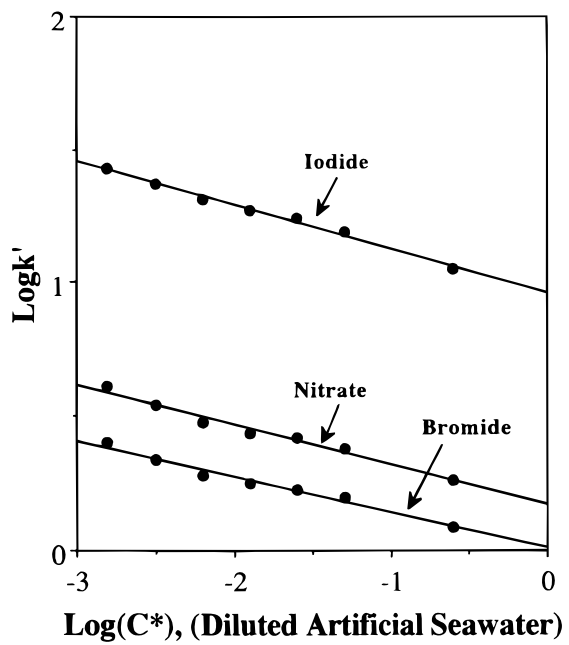

Figure 2. $\log K$ versus $\log \left(C^{\star}\right)$, where $C^{\star}$ is the dilution factor of artificial seawater used as eluent.

monovalent analyte anions eluted via an ion exchange mechanism using a monovalent eluent. Even allowing for the presence of some divalent eluent anions (sulfate), the observed slopes show that a conventional ion-exchange mechanism is not being followed. The different retention behavior evident between Figures 1 and 2 is attributable to the fact that the eluent in Figure 2 contains a mixture of ions. Other eluents containing more than one ionic salt were therefore prepared and used as eluents for the separation of the same sample. The experimental results showed that when a divalent cation $\left(\mathrm{Ca}^{2+}\right.$ and/ or $\mathrm{M} \mathrm{g}^{2+}$ ) and $\mathrm{SO}_{4}{ }^{2-}$ were simultaneously present in the eluent, $\log \mathrm{K}^{\prime}$ always decreased slightly in a linear manner with increasing log [eluent].

Proposed Mechanism. For a zwitterionic surfactant stationary phase conditioned with an electrolytic eluent, a "binary" electrical double layer (EDL) can be expected to be created. That is, the eluent cations build up an EDL around the negatively charged (sulfonate) functional groups on the surfactant, while the eluent anions form an EDL around the positively charged (quaternary ammonium) functional groups. This is illustrated in Figure 3 for the case used in this experiment, that is, for a zwitterionic surfactant in which the sulfonate group is terminal on the molecule and can therefore be expected to be situated furthest from the ODS surface. In this type of EDL, the cations and anions are present in equimolar amounts. When a sample is injected onto the binary EDL stationary phase, the sample cations should show no retention due to repulsion by the cationic layer situated at the top part of the binary EDL. In contrast, analyte anions can be expected to show retention because they are able to enter into the binary EDL. Further influences on the mechanism of retention of the sample anions by the binary EDL can be explained by using the concept of ion-pair formation.

The concept of ion-pair formation was initially suggested by Bjerrum in 1926,20 who proposed that in an electrolytic solution the Coulombic attraction between ions of opposite charges could temporarily overcome the thermal energy that tends to separate ions after they have been hydrated in aqueous solutions, leading to the formation of an association between the cation and anion, or an ion pair. To date, the existence of ion pairs has been widely accepted and has been used to specify many of the properties of electrolytic solutions. ${ }^{21-23}$ Sample anions distributed into the

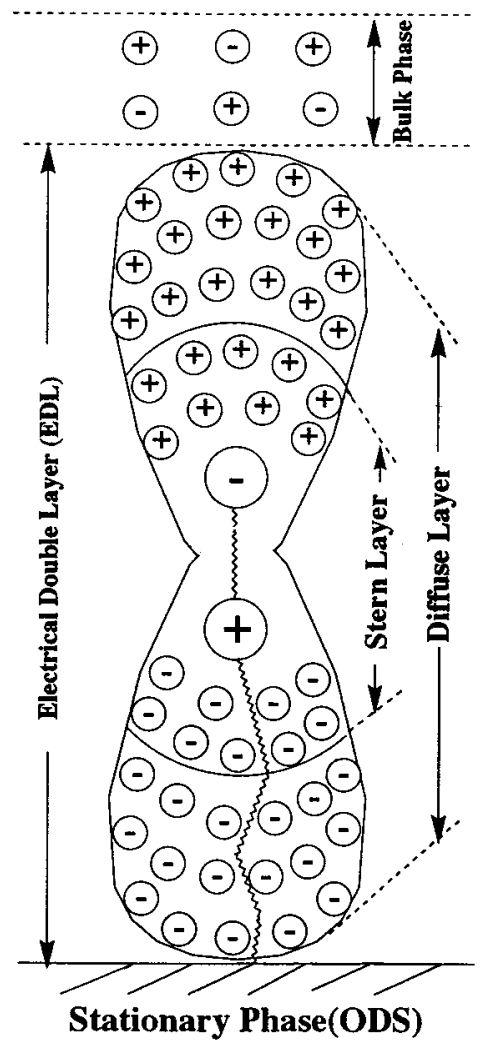

Figure 3. Schematic diagram (not drawn to scale) of the adsorbed surfactant showing the binary electrical double layer established by eluent cations retained by the negatively charged sulfonate functional groups of the zwitterionic surfactant and eluent anions retained by the positively charged quaternary ammonium functional groups of the zwitterionic surfactant.

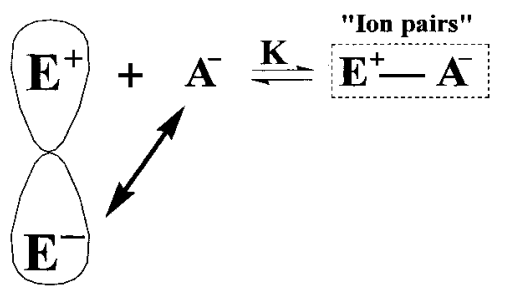

Figure 4. Schematic representation of the distribution of analyte anions into the cation-EDL followed by retention due to the formation of neutral ion pairs with the EDL cation and simultaneous repulsion from the anionic EDL.

cationic outer layer of the binary EDL surrounding the sulfonate functional group can be expected to participate in the formation of neutral ion pairs, but at the same time should experience repulsion by the anionic EDL surrounding the quaternary ammonium functional group (Figure 4). Therefore, sample anions that have a high propensity to form neutral ion pairs with the EDL cations should show strong retention, while those with a low propensity to form ion pairs should be expelled from the EDL and therefore show little or no retention. Thus, the value of the association constant for the neutral ion pair should be a good indicator for predicting the extent of retention of the sample anions. Of the analytes tested, iodide was eluted with the longest retention time, followed by nitrate, and then bromide. This order of retention is found to be in order of increasing ionic size, increasing polarizability, and decreasing hydration energy of the sample anions. It should be noted here that under circumstances 
where the ion-pair formation is very strong (such as for very polarizable anions) elution from the stationary phase may not occur because of the inadequacy of the repulsion by the anionic EDL to eject the anions from the EDL. Sulfate shows no retention in this system, which can be attributed either to its low propensity to form ion pairs or to the formation of an anionic ion pair [ $\mathrm{Na}^{+}-$ $\left.\mathrm{SO}_{4}{ }^{2-}\right]^{-}$, with subsequent repulsion by the anionic EDL.

Once the binary EDL depicted in Figure 3 has been established, further addition of eluent ions should show very little effect on the retention of the analyte anions, in accordance with the experimental results shown in Figure 1. On the other hand, maintenance of the binary EDL should require the concentration of the electrolyte in the eluent to be sufficiently high $(5.0 \mathrm{mM}$ was found to be sufficient for this purpose).

Turning now to the retention behavior exhibited using eluents comprising mixed electrolytes containing $\mathrm{Ca}^{2+}$ and/ or $\mathrm{M} \mathrm{g}^{2+}$ and $\mathrm{SO}_{4}{ }^{2-}$ (Figure 2), it is apparent that there is a further factor influencing retention since increased eluent concentration now causes reduced retention of analyte anions. This behavior, together with a linear relationship between $\log k^{\prime}$ and $\log$ [eluent], is characteristic of ion exchange. One possible explanation is that $\mathrm{Ca}^{2+}$ and/ or $\mathrm{M} \mathrm{g}^{2+}$ ions would be incorporated into the cationic component of the EDL and retention would be dependent on the ability of the analyte anion to form ion pairs with these cations. On the other hand, $\mathrm{SO}_{4}{ }^{2-}$ is known to form neutral ion pairs with $\mathrm{Ca}^{2+}$ and/ or $\mathrm{M} \mathrm{g}^{2+}$, so this ion could be envisaged to compete with analyte anions for ion-pair formation in the cationic EDL. Increasing the concentration of $\mathrm{SO}_{4}{ }^{2-}$ in the eluent would lead to increased competition and thereby reduced retention of the analyte anion, as observed in Figure 2.

Analysis of Seawater Samples. The standard sample was analyzed 20 times using the 20-fold-diluted artificial seawater as the eluent, and the relative standard deviations were found to be better than $1.2 \%$ for the retention times, peak areas, and peak heights for all of the analytes. The detection limit with UV-visible at $210 \mathrm{~nm}$ was found to be $0.75 \mathrm{ppb}$ for bromide, $0.52 \mathrm{ppb}$ for nitrate, and $0.8 \mathrm{ppb}$ for iodide using an injection volume of 100 $\mu \mathrm{L}$, indicating that the method was sufficiently sensitive for the direct determination of these species in most types of natural seawater. Recoveries of added $\mathrm{Br}^{-}(500 \mathrm{ppb}), \mathrm{NO}_{3}{ }^{-}(50 \mathrm{ppb})$, and $\mathrm{I}^{-}(5.0 \mathrm{ppb})$ were $99.75,99.43$, and $99.12 \%$ respectively.

Real seawater was analyzed by direct injection, and Figure 5 shows the chromatogram obtained using 20 -fold-diluted artificial seawater as the eluent. A chromatogram of the standard sample is also included in Figure 5 for comparison. The concentrations of bromide, nitrate, and iodide in this seawater sample were found to be $4.61 \mathrm{ppm}, 48.8 \mathrm{ppb}$, and $5.4 \mathrm{ppb}$, respectively. The method described in this paper can be seen to be applicable to the direct determination of bromide, nitrate, and iodide in seawater. Both the standard saline sample and the real seawater sample were also analyzed using $10.0 \mathrm{mM} \mathrm{Na}_{2} \mathrm{SO}_{4}$ or $10.0 \mathrm{mM} \mathrm{NaCl}$ as the eluent, and good separations were also achieved. However, these eluents have the disadvantage that changes in eluent concentration do not lead to significant changes in retention times, thereby making it difficult to optimize a separation.

The retention of nitrite has also been studied, and nitrite can be completely separated from bromide, nitrate, and iodide. However, when applied to the analysis of the seawater sample,

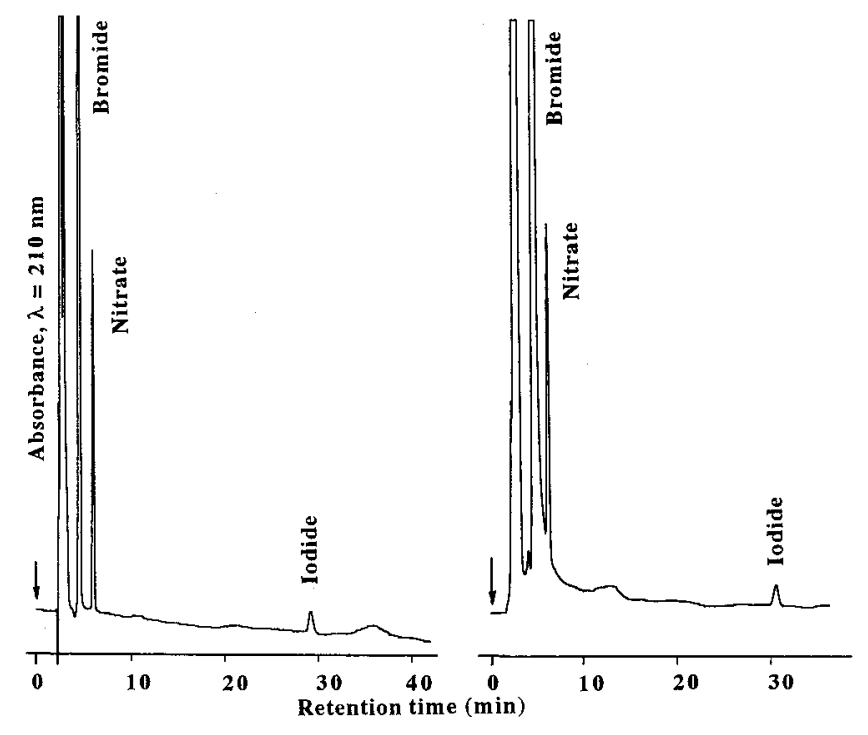

Figure 5. Chromatograms of a standard saline sample (left-hand trace) $\left(\mathrm{Br}^{-}, 1000 \mathrm{ppb} ; \mathrm{NO}_{3}{ }^{-}, 50 \mathrm{ppb}\right.$; and $\mathrm{I}^{-}, 5.0 \mathrm{ppb}$, dissolved in the artificial seawater matrix) and an actual seawater sample (righthand trace) obtained using a 20 -fold-diluted artificial seawater as the eluent. Column, ODS-packed column $(250 \times 4.6$, id., $\mathrm{mm})$ coated with Zwittergent-3-14 micelles; flow rate, $1.0 \mathrm{~mL} / \mathrm{min}$; sample injection volume, $100 \mu \mathrm{L}$; detection, UV-visible at $210 \mathrm{~nm}$.

the nitrite peak is obscured by the first large peak in the chromatogram (Figure 5) which arises from the elution of the matrix ions. The stationary phase (ODS modified with zwitterionic surfactant) was found to exhibit excellent stability and reproducibility, with the same column giving relative standard deviations of $<1.2 \%$ for the retention times, peak areas, and peak heights for bromide, nitrate, and iodide, over a 3-month period when operated at $30^{\circ} \mathrm{C}$.

\section{CONCLUSIONS}

The combination of a zwitterionic stationary phase and a dilute electrolytic eluent has been shown to be useful for the separation of UV-absorbing analyte anions in a saline matrix. Retention times can be manipulated by varying the concentration of the electrolyte in the eluent, provided the eluent contains $\mathrm{Ca}^{2+}$ and/ or $\mathrm{M} \mathrm{g}^{2+}$ and $\mathrm{SO}_{4}{ }^{2-}$. A retention mechanism has been proposed which involves the initial establishment of a binary EDL at the two functional groups of the zwitterionic stationary phase, followed by incorporation of analyte anions into the cationic component of the binary $E D L$ and subsequent competiton between ion-pairing effects and repulsion by the anionic component of the binary EDL. Analyte anions that have the highest propensity to form ion pairs with the eluent cations show the strongest retention. This method is applicable to the direct determination of UV-absorbing anions in seawater (or other type of saline samples). It would be very interesting to investigate the possibility of separation of cations by creation of a binary EDL having a configuration opposite to that used in this study (i.e., the anion-EDL being outermost, and the cation-EDL being innermost). Such a binary EDL could be created using a phosphocholine type of zwitterionic surfactant as the stationary phase.

Received for review August 4, 1998. Accepted January 8, 1999.

\section{AC980860M}

